\title{
MicroRNA 28-5p regulates ATP-binding cassette transporter A1 via inhibiting extracellular signal-regulated kinase 2
}

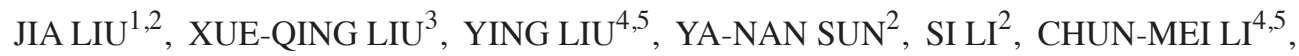 \\ $\mathrm{JIE} \mathrm{LI}^{4,5}$, WEI TIAN ${ }^{4,5}$, XIAO-MING SHANG ${ }^{1,2}$ and YUN-TAO ZHOU ${ }^{4,5}$ \\ ${ }^{1}$ Department of Internal Medicine, Hebei Medical University, Shijiazhuang, Hebei 050017; ${ }^{2}$ Department of \\ Cardiology, Tangshan Gongren Hospital, Tangshan, Hebei 063000; ${ }^{3}$ Department of Hepatobiliary Surgery, \\ The Second Hospital of Hebei Medical University, Shijiazhuang, Hebei 050000; ${ }^{4}$ Department of \\ Clinical Laboratory Center; ${ }^{5}$ Tangshan Key Laboratory of Clinical Molecular Diagnosis \\ and Treatment, Tangshan Gongren Hospital, Tangshan, Hebei 063000, P.R. China
}

Received November 4, 2014; Accepted July 29, 2015

DOI: $10.3892 / \mathrm{mmr} .2015 .4563$

\begin{abstract}
The biological function of the intronic microRNA-28 (miR-28) may be associated with the biological roles of its host gene, LIM domain lipoma-preferred partner (LPP). LPP has been reported to promote smooth muscle cell migration in arterial injury and atherosclerosis. However, the mechanism of miR-28 in atherosclerosis remains unclear. In the current study, the aim was to validate the inhibitory effect of miR-28-5p on extracellular signal-regulated kinase 2 (ERK2), to investigate its biological role in atherosclerosis and its association with cardiovascular disease. Western blotting and stem-loop reverse transcription-quantitative polymerase chain reaction combined with TaqMAN microRNA analysis was conducted. The current study demonstrated that miR-28-5p upregulated the expression of ATP-binding cassette transporter A1 (ABCA1) via the inhibition of ERK2 in HepG2 cells. In addition, increased levels of plasma miR-28-5p were positively correlated with the levels of high-density lipoprotein cholesterol in patients with unstable angina. This suggests that miR-28-5p participates in atherosclerosis via ERK2-mediated upregulation of the ABCA1 pathway.
\end{abstract}

Correspondence to: Professor Yun-Tao Zhou, Department of Clinical Laboratory Center, Tangshan Gongren Hospital, 27 Wenhua Road, Tangshan, Hebei 063000, P.R. China

E-mail: yuntaozhou@hotmail.com

Abbreviations: LPP, lipoma-preferred partner; ABCA1, ATP-binding cassette transporter A1; ERK2, extracellular signal-regulated kinase 2; UA, unstable angina; UTR, untranslated region; HDL-C, high-density lipoprotein cholesterol; LDL-C, low-density lipoprotein cholesterol; Glu, glucosamine; CHOL, cholesterol; TG, triglyceride; APOA1, apolipoprotein A1; LP( $\alpha$, lipoprotein $\alpha$; CK-MB, creatine kinase-myocardial band

Key words: miR-28-5p, ERK2, ABCA1, unstable angina

\section{Introduction}

MicroRNAs (miRNAs) are endogenous non-coding RNAs and are key post-transcriptional regulators, inhibiting the translation or promoting the degradation of target mRNAs via binding to the 3' untranslated regions (UTRs) (1). These miRNAs have been identified to be involved in complex physiological processes including differentiation, proliferation and metabolism (2). Previous studies suggest that intronic miRNAs, which are located in the intron regions of host genes, may construct a fine-tuning regulatory system with their host genes and trigger cell transition by responding to external stimuli $(3,4)$. The gene encoding LIM domain lipoma-preferred partner (LPP) has been reported to promote smooth muscle cell (SMC) migration in arterial injury and atherosclerosis (5-7), whereas its intronic miRNA, miRNA-28 (miR-28), is associated with tumor cell migration, adhesion, proliferation and apoptosis $(8,9)$. A previous preliminary study (10) indicated that the transfection of miR-28-5p mimics increased the expression of ABCA1 in HepG2 cells and THP-1-derived macrophages. In addition, the circulating levels of miR-28-5p were significantly increased in a relatively small sample population of patients with unstable angina (UA). Therefore, investigation of miR-28 may provide insight into the cholesterol metabolism in atherosclerosis.

Extracellular signal-regulated kinase 2 (ERK2) is a member of a highly conserved family of serine-threonine protein kinases and is a key regulator in cell growth and differentiation (11). ERK2 is activated or overexpressed in numerous types of cancer, and may be a potential therapeutic target (12). In addition, ERK2 has been demonstrated to be involved in cardiac development and protection (13). Zhou et al (14) reported a novel role for ERK2 activity in cholesterol trafficking, demonstrating that inhibition of ERK1/2 markedly increases ATP-binding cassette transporter A1 (ABCA1) expression and leads to increased cholesterol efflux in macrophages. $\mathrm{ABCA} 1$ is a key mediator in the maintenance of high-density lipoprotein cholesterol (HDL-C) biosynthesis in the liver and cholesterol efflux in macrophages $(15,16)$. Mutations in the ABCA1 gene lead to Tangier disease, which is characterized by impaired cholesterol efflux and low levels of HDL, 
leading to an increased risk of atherosclerotic disease (17-19). Due to the anti-atherogenic properties of ABCA1 and ERK2, they represent potential targets through which atherosclerosis may be attenuated via ABCA1 upregulation. miRWalk target prediction previously indicated that miR-28-5p is the highest ranking miRNA predicted to target ERK2 (20), suggesting that it may regulate ABCA1 expression via ERK2 inhibition.

Girardot et al (21) validated the fact that ERK2 is an miR-28-targeted gene using the psi-CHECK-2 luciferase reporter system in the Mo7e human megakaryoblastic leukemia cell line. In the current study, the inhibitory effect on ERK2 was investigated via the transfection of miR-28-5p mimics and inhibitors into HepG2 cells and the assessment of the mechanism of miR-28-5p-mediated ABCA1 upregulation. Additionally, the association between the plasma levels of miR-28-5p and HDL-C levels was evaluated in a sample population of patients with UA to investigate its potential use as a biomarker and as a therapeutic target in atherosclerosis.

\section{Materials and methods}

Cell culture and transfection. The HepG2 human hepatoma cell line was obtained from the Cell Culture Engineering Center of the Chinese Academy of Medical Sciences and Peking Union Medical College (Beijing, China). HepG2 cells were cultured in 6 well plates with $2.0 \mathrm{ml} /$ well Dulbecco's modified Eagle's medium supplemented with $10 \%$ heat-inactivated fetal bovine serum (Invitrogen Life Technologies, Carlsbad, CA, USA), $100 \mathrm{U} / \mathrm{ml}$ penicillin and $100 \mu \mathrm{g} / \mathrm{ml}$ streptomycin (Invitrogen Life Technologies) at $37^{\circ} \mathrm{C}$ in a $5 \% \mathrm{CO}_{2}$ incubator. HepG2 cells were serum-starved for a minimum of $16 \mathrm{~h}$ prior to treatment.

Control mimics, miR-28-5p mimics or inhibitors (100 nM; Invitrogen Life Technologies) were transiently transfected into HepG2 cells using Lipofectamine ${ }^{\circledR}$ RNAi 2000 Reagent and Opti-MEM I Reduced Serum Medium (Invitrogen Life Technologies) according to the manufacturer's instructions. Cells were treated with the ERK2 inhibitor PD98059 (Santa Cruz Biotechnology, Inc., Dallas, TX, USA) as described previously (13). Briefly, HepG2 cells were pre-transfected with or without the mir-28-5p inhibitor and incubated with 20 or $40 \mu \mathrm{M}$ PD98059 overnight. Following $48 \mathrm{~h}$ of transfection, cells were harvested for protein analysis or were treated for further experiments. Briefly, the treated cells were washed with times with phosphate-buffered saline, and lysed by radioimmunoprecipitation acid buffer (Beyotime Institute of Biotechnology, Shanghai, China) at $4^{\circ} \mathrm{C}$. All transfection experiments were performed at least three times.

Western blotting. Treated cells were lysed in radioimmunoprecipitation assay lysis buffer (Beyotime Institute of Biotechnology). Protein concentrations were determined using a Bicinchoninic Acid Protein Assay kit (Beyotime Institute of Biotechnology). Protein extracts (40 $\mu \mathrm{g} / \mathrm{lane})$ were separated on a $10 \%$ SDS-polyacrylamide gel (Sigma-Aldrich, St. Louis, MO, USA) and transferred to polyvinylidene difluoride membranes (EMD Millipore, Billerica, MA, USA) using a Mini Trans-Blot ${ }^{\circledR}$ system (Bio-Rad Laboratories, Inc., Shanghai, China). Membranes were blocked with immuno-blotting blocking buffer (Beyotime Institute of Biotechnology) for $1 \mathrm{~h}$ at room temperature prior to incubation with the following primary antibodies obtained from Santa Cruz Biotechnology, Inc.: Polyclonal rabbit anti-ABCA1 (1:500; cat no. sc-20794), polyclonal rabbit anti-ERK2 (1:500; cat no. sc-154) or polyclonal rabbit anti- $\beta$-actin $(1: 1,000$; cat no. sc-130656) overnight at $4^{\circ} \mathrm{C}$. Following washing with Tris-buffered saline with Tween-20, the membranes were incubated for $2 \mathrm{~h}$ at room temperature with horseradish peroxidase-conjugated goat anti-rabbit IgG antibodies (1:5,000; cat no. sc-2004; Santa Cruz Biotechnology, Inc.). Protein bands were visualized by chemiluminescence using the SuperSignal ${ }^{\mathrm{TM}}$ West Dura Substrate (Thermo Fisher Scientific, Waltham, MA, USA). Signal intensity was quantified using AlphaEaseFC 4.0 software (Cell Biosciences, Inc., Santa Clara, CA, USA) and normalized to $\beta$-actin levels.

Study population. All patients with UA $(n=39)$ in the current study were enrolled from the Department of Cardiology in Tangshan Gongren Hospital (Tangshan, China) between March 2012 and August 2013. The diagnosis of UA was confirmed using the American College of Cardiology and the American Heart Association 2007 guidelines (22). Healthy subjects $(n=28)$ were selected if the following conditions were absent: Diabetes, hypertension, hepatitis, kidney disease, cardiovascular disease and medication history at the Tangshan Gongren Hospital. The current study was approved by the Ethics Committee of Tangshan Gongren Hospital and written informed consent was obtained from each volunteer.

Clinical samples. The handling of blood samples was completed within $2 \mathrm{~h}$ of collection according to the Tangshan Gongren Hospital Biobank Handling and Storage Protocol for the collection, processing and archiving of human blood. Briefly, fasting blood samples were drawn in the morning and collected into EDTA anticoagulant tubes (Guangzhou Improve Medical Instruments Co., Ltd., Guangzhou, China) and centrifuged at $3,000 \mathrm{x} \mathrm{g}$ then $16,000 \mathrm{x} \mathrm{g}$ for $10 \mathrm{~min}$ at $4^{\circ} \mathrm{C}$ each to separate the plasma from blood cells, platelets and cellular debris. Plasma was then transferred to RNase/DNase-free $1.5 \mathrm{ml}$ Cryo tubes (Sangon Biotech Co., Ltd., Shanghai, China) and stored at $-80^{\circ} \mathrm{C}$ until required.

$R N A$ isolation and reverse transcription-quantitative polymerase chain reaction ( $R T-q P C R)$. Total small RNAs were isolated from $500 \mu \mathrm{l}$ plasma using the mirVana ${ }^{\mathrm{TM}}$ RNA Isolation kit (Ambion Life Technologies, Carlsbad, CA, USA) according to the manufacturer's instructions. Extracted RNAs were eluted with $80 \mu \mathrm{l}$ diethylpyrocarbonate-treated $\mathrm{ddH}_{2} \mathrm{O}$ (Sangon Biotech Co., Ltd.) and stored at $-80^{\circ} \mathrm{C}$. Quality of RNA was determined using a NanoDrop ${ }^{\mathrm{TM}} 2000$ Spectrophotometer (Thermo Fisher Scientific).

The expression levels of miR-28-5p and miR-423-3p were determined by RT-qPCR. Briefly, small RNA was reverse transcribed into DNA using the TaqMan ${ }^{\circledR}$ MicroRNA Reverse Transcription kit (Applied Biosystems Life Technologies, Foster City, CA, USA). In brief, the RT reaction mixture contained $0.8 \mu 1$ 10X reverse transcription buffer, $4 \mu \mathrm{l}$ small RNA ( 20 ng/ $\mu \mathrm{l}), 0.1 \mu 1100 \mathrm{mM}$ dNTPs, $0.1 \mu \mathrm{l}$ RNase inhibitor $(20 \mathrm{U} / \mu 1), 1.5 \mu 1 \mathrm{RT}$ primer, $0.5 \mu 1$ MultiScribe ${ }^{\mathrm{TM}}$ Reverse Transcriptase $(50 \mathrm{U} / \mu \mathrm{l})$ and $1 \mu \mathrm{l}$ Rase/DNase-free $\mathrm{ddH}_{2} \mathrm{O}$. 
The reaction mixture was incubated at $16^{\circ} \mathrm{C}$ for $60 \mathrm{~min}, 42^{\circ} \mathrm{C}$ for $60 \mathrm{~min}$ and $85^{\circ} \mathrm{C}$ for $5 \mathrm{~min}$. RT-qPCR was performed using an Applied Biosystems ${ }^{\circledR} 7500$ Real-time PCR System (Applied Biosystems Life Technologies). The PCR reaction mixture contained $4 \mu \mathrm{l}$ RT product, $10 \mu 1$ 2X TaqMan Universal PCR Master Mix, $1 \mu 1$ TaqMan probe $(2.5 \mu \mathrm{M})$ and $5 \mu \mathrm{l}$ RNase/DNase-free $\mathrm{ddH}_{2} \mathrm{O}$. The cycling parameters were as follows: Denaturation at $95^{\circ} \mathrm{C}$ for $10 \mathrm{~min}$ followed by 40 cycles each of denaturation at $95^{\circ} \mathrm{C}$ for $15 \mathrm{sec}$ and annealing and extension at $60^{\circ} \mathrm{C}$ for $1 \mathrm{~min}$. miRNA levels were determined using the 2-[Ct(target miRNA)-Ct(reference miRNA)] method. hsa-miR-423 was used as a stable internal reference in the plasma samples according to the guidelines of $\mathrm{ABI}$ miRNA profiling (Applied Biosystems Life Technologies) in serum/plasma and as previously reported (23).

miRNA target prediction. miRNA target interactions were predicted using the miRwalk database (http://www.umm. uni-heidelberg.de/apps/zmf/mirwalk). miRNA sets for ERK2 were obtained based on the miRwalk prediction algorithm.

Statistical analysis. Data were presented as the mean \pm standard error unless otherwise stated. Comparisons between two groups were performed using the Mann-Whitney U-test or Student's t-test as appropriate. A Fisher's exact test or $\chi^{2}$ test was used to compare categorical variables. Correlation analysis was performed using Spearman's correlation analysis. Statistical analysis was performed with GraphPad Prism software, version 5.0 (GraphPad Software, Inc., La Jolla, CA, USA). P<0.05 was considered to indicate a statistically significant difference.

\section{Results}

Identification of the target gene of $\mathrm{miR}-28-5 \mathrm{p}$ in Hep $\mathrm{G} 2 \mathrm{cells}$. To validate the fact that miR-28-5p targets ERK2, the protein levels of ERK2 in HepG2 cells were measured following transfection with the miR-28-5p mimic or inhibitor. Western blotting indicated that transfection with the miR-28-5p mimic significantly reduced the expression levels of ERK2 in HepG2 cells, whereas the miR-28-5p inhibitor resulted in elevated levels of ERK2 following $48 \mathrm{~h}$ of transfection (Fig. 1). These results indicate that miR-28-5p is a key mediator in the regulation of the translation of ERK2.

Effect of miR-28-5p mimics or inhibitors on ABCA1 expression in HepG2 cells. In a previous preliminary study (10), the transfection of miR-28-5p mimics was observed to result in an increase in ABCA1 expression levels in HepG2 cells and THP-1-derived macrophages. In the current study, miR-28-5p inhibitors were transfected into HepG2 cells to further investigate the miR-28-5p-mediated upregulation of ABCA1. As presented in Fig. 2, transfection with the miR-28-5p inhibitor significantly reduced the expression levels of ABCA1 $(\mathrm{P}<0.05)$ compared with control and miR-28-5p mimics (Fig. 2).

miR-28-5p upregulates ABCAl expression through the inhibition of ERK2. To investigate whether ERK2 alters the expression of ABCA1, HepG2 cells were incubated
A

\section{HepG2}

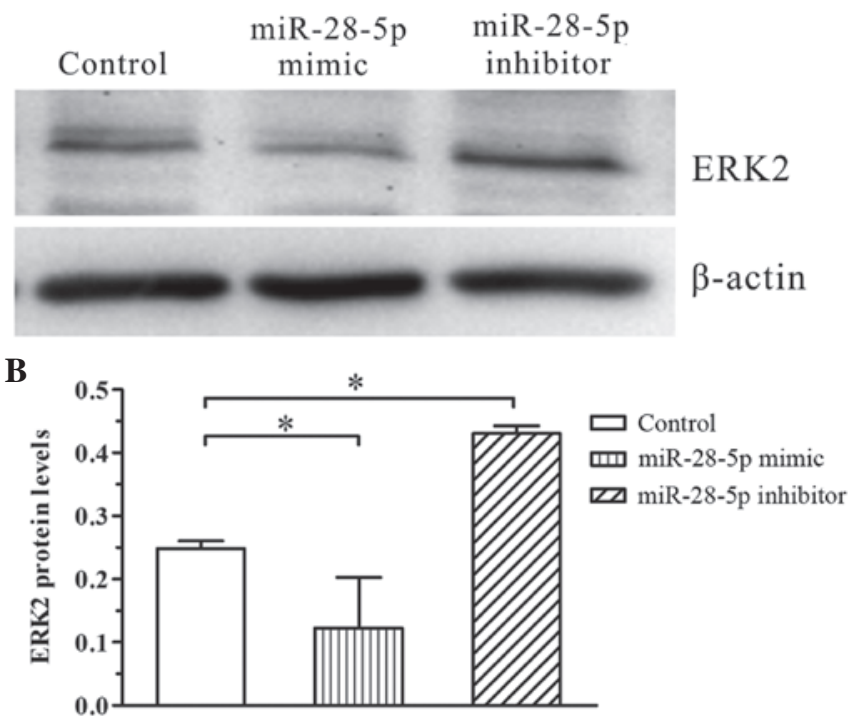

Figure 1. Identification of the target gene of miR-28-5p in HepG2 cells (A) Western blotting was used to identify the target gene of miR-28-5p in HepG2 cells. (B) Quantification indicates that the protein level of ERK2 was significantly reduced in HepG2 cells transfected with the miR-28-5p mimic, compared with the control group. The expression level of ERK2 was increased in HepG2 cells transfected with the miR-28-5p inhibitor. " $\mathrm{P}<0.05$. miR-28-5p, microRNA-28-5p; ERK2, extracellular signal-regulated kinase 2.

A

\section{HepG2}

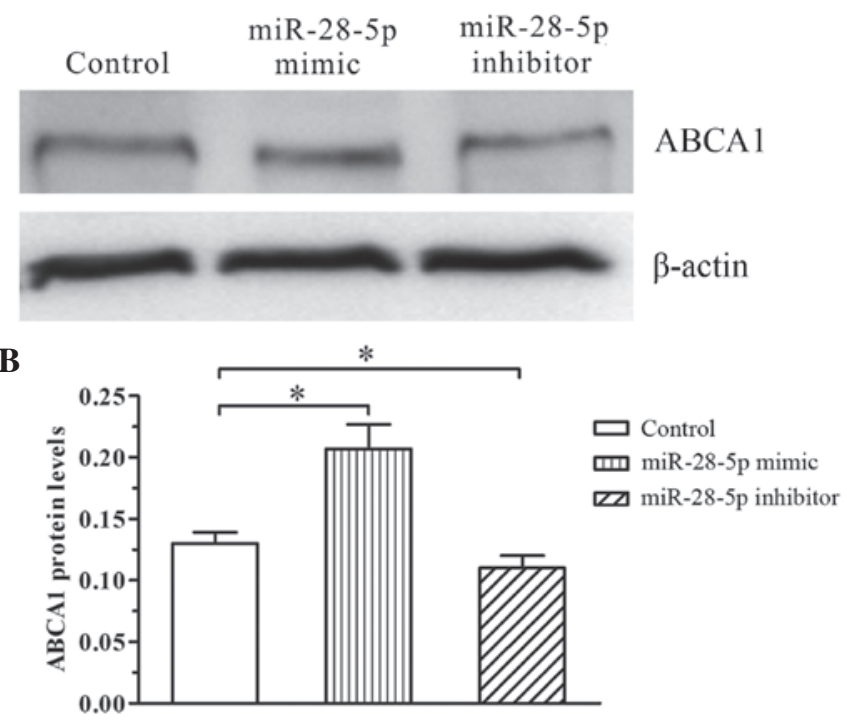

Figure 2. miR-28-5p increases ABCA1 protein level. (A) Western blot analysis of ABCA1 in HepG2 cells transfected with the miR-28-5p mimic or inhibitor. (B) Quantification demonstrated that overexpression of miR-28-5p following transfection with its mimic resulted in increased levels of ABCA1 protein in HepG2 cells. The transfection with the miR-28-5p inhibitor significantly reduced ABCA1 expression levels. These results indicate that miR-28-5p is a key mediator for regulating the translation of ABCA1. ${ }^{*} \mathrm{P}<0.05$. miR-28-5p, microRNA-28-5p; ABCA1, ATP-binding cassette transporter A1.

with an ERK2 inhibitor, PD98059. As previously reported, the induction of ABCA1 expression is semi-dependent on the concentration of ERK 2 inhibitors, with the maximal induction effect dose for macrophage ABCA1 expression by 
Table I. Clinical characteristics of subjects between the control and UA groups.

\begin{tabular}{|c|c|c|c|}
\hline Characteristic & $\begin{array}{l}\text { Control } \\
(n=28)\end{array}$ & $\begin{array}{c}\text { UA } \\
(n=39)\end{array}$ & P-value \\
\hline Age (years) & $55 \pm 8$ & $58 \pm 9$ & 0.291 \\
\hline Gender (male/female) & $13 / 15$ & $21 / 18$ & 0.624 \\
\hline Glu (mmol/l) & $5.36 \pm 0.52$ & $5.33 \pm 1.39$ & 0.959 \\
\hline CHOL (mmol/l) & $5.25 \pm 095$ & $4.18 \pm 0.97$ & $<0.001$ \\
\hline $\mathrm{TG}(\mathrm{mmol} / \mathrm{l})$ & $1.31 \pm 0.62$ & $1.12 \pm 0.34$ & 0.116 \\
\hline HDL-C (mmol/l) & $1.19 \pm 0.24$ & $1.02 \pm 0.189$ & 0.002 \\
\hline LDL-C (mmol/l) & $3.00 \pm 0.81$ & $2.65 \pm 0.93$ & 0.115 \\
\hline APOA1 (g/l) & - & $1.13 \pm 0.19$ & - \\
\hline APOB100 (g/l) & - & $0.80 \pm 0.17$ & - \\
\hline $\mathrm{LP}(\alpha)(\mathrm{mg} / \mathrm{l})$ & - & $316.46 \pm 247.75$ & - \\
\hline CK-MB & - & $13.11 \pm 5.75$ & - \\
\hline Smokers & $13 / 15$ & $21 / 18$ & 0.624 \\
\hline Hypertension & none & none & - \\
\hline Diabetes mellitus, n (\%) & none & 31.58 & - \\
\hline
\end{tabular}

Dash indicates the value was not measured; none indicates no history of hypertension or diabetes. UA, unstable angina; Glu, glucosamine; CHOL, cholesterol; TG, triglyceride; HDL-C, high-density lipoprotein cholesterol; LDL-C, low-density lipoprotein cholesterol; APOA1, apolipoprotein A1; APOB100, apolipoprotein B100; LP( $\alpha$ ), lipoprotein $\alpha$; CK-MB, creatine kinase myocardial band.

A

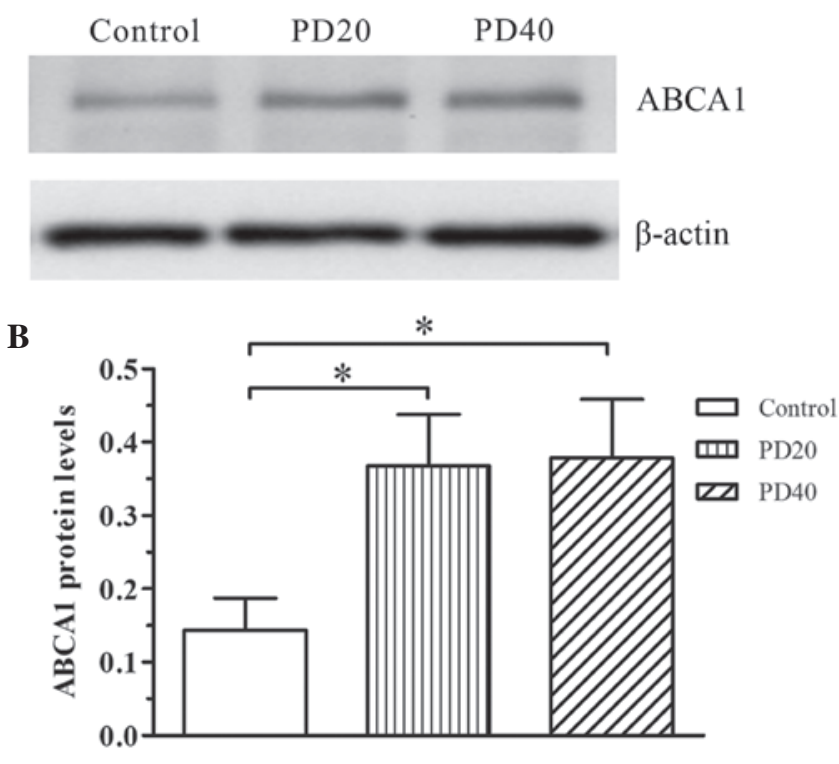

Figure 3. ERK2 inhibitor (PD98059) increases expression levels of ABCA1. (A) Western blot analysis of ABCA1 in HepG2 cells incubated with 20 or $40 \mu \mathrm{M}$ ERK2 inhibitor (PD98059). (B) Quantification indicated that PD98059 increased the expression levels of ABCA1 in HepG2 cells. ${ }^{*} \mathrm{P}<0.05$. ERK2, extracellular signal-regulated kinase 2; ABCA1, ATP-binding cassette transporter A1; PD20, $20 \mu \mathrm{M}$ PD98059; PD40, $40 \mu \mathrm{M}$ PD98059.

PD98059 being $20 \mu \mathrm{M}$ (14). As presented in Fig. 3, PD98059 increased the expression levels of ABCA1 in HepG2 cells.

To confirm that the miR-28-5p-mediated upregulation of ABCA1 is dependent on ERK2, HepG2 cells were transfected with the miR-28-5p inhibitor prior to incubation with the ERK2 inhibitor (PD98059) overnight. As presented in Fig. 4,
HepG2 cells stimulated with $20 \mu \mathrm{M}$ PD98059 antagonized the miR-28-5p inhibitor-mediated ABCA1 reduction. Taken together, these data indicate that miR-28-5p mediates ABCA1 expression through the ERK2 signaling pathway.

To explore the potential miRNAs with the ability to upregulate ABCA1 through ERK2 inhibition, the predicted dataset of miRNA and ERK2 interactions were retrieved from the miRwalk database. The top 30 predicted miRNAs are shown as Table II, and all have potential roles in ERK2-mediated ABCA1 upregulation.

Circulating levels of $\mathrm{miR}-28-5$ p positively correlate with $H D L-C$ levels. Circulating levels of miR-28-5p in healthy subjects $(n=28)$ and patients with UA $(n=39)$ were measured to investigate the association between the circulating levels of miR-28-5p and clinical parameters in patients with UA. Clinical parameters of patients with UA are presented in Table I. There were no significant differences between the groups in age, gender or risk factors except for total cholesterol and HDL-C. The circulating levels of miR-28-5p were not significantly associated with age or gender or with diabetic status. However, the circulating levels of miR-28-5p in patients with UA were observed to be positively correlated with HDL-C levels ( $\mathrm{R}=-0.327$; $\mathrm{P}=0.042$; Fig. 5), supporting the role of miR-28-5p in the upregulation of ABCA1 in vitro.

\section{Discussion}

A previous study indicated that miR-28-5p inhibits the expression of LPP (24), which is involved in SMC phenotype transition in atherosclerosis $(6,7)$, suggesting that it may serve a potential role in cardiovascular disorders. A previous preliminary study (10) demonstrated that miR-28-5p mimics 


\section{HepG2}
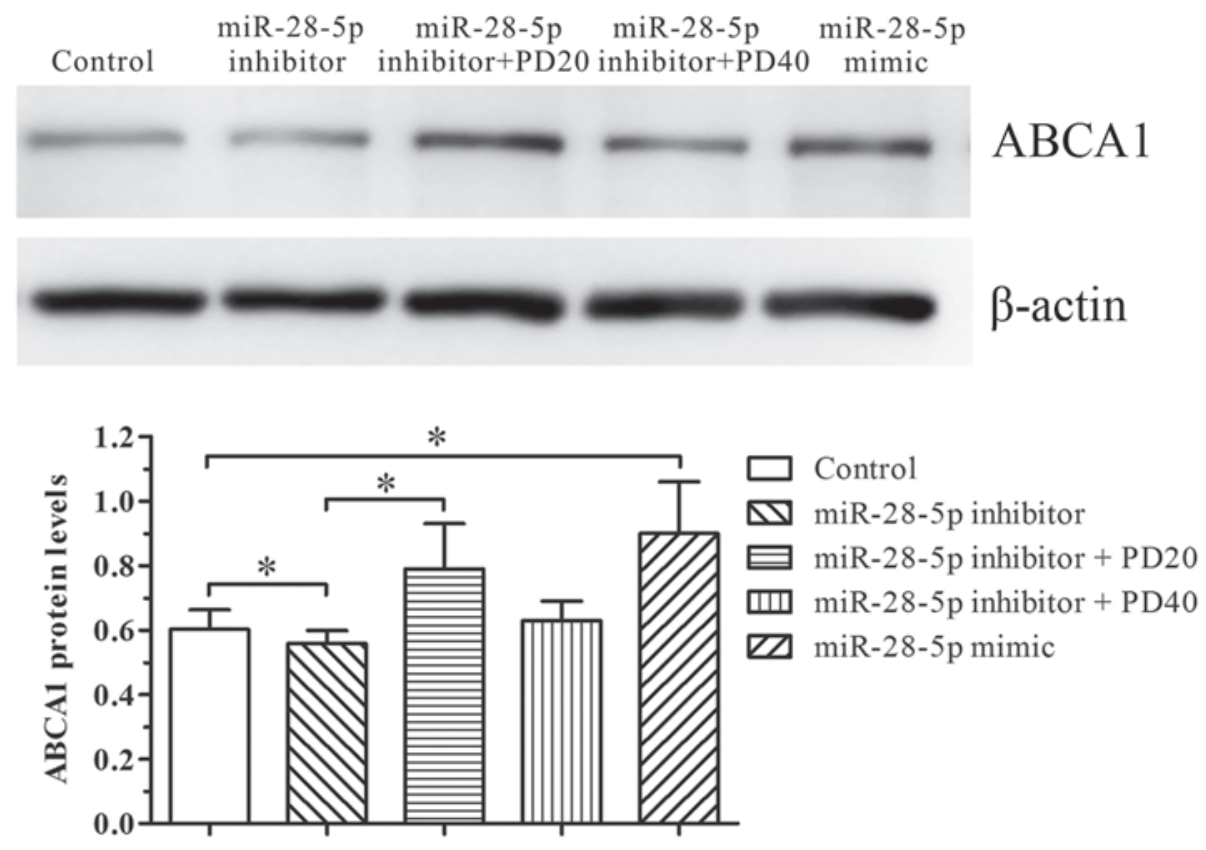

Figure 4. miR-28-5p increases ABCA1 expression via ERK2. Transfection with the miR-28-5p inhibitor or mimic resulted in reduced or increased protein expression levels of ABCA1, respectively, compared with the control group. miR-28-5p inhibitor transfection of HepG2 cells incubated with 20 or $40 \mu \mathrm{M}$ ERK2 inhibitor (PD98059) increased or restored ABCA1 expression levels, respectively. "P<0.05. miR-28-5p, microRNA-28-5p; ABCA1, ATP-binding cassette transporter A1; ERK2, extracellular signal-regulated kinase 2; PD20, $20 \mu \mathrm{M}$ PD98059; PD40, $40 \mu \mathrm{M}$ PD98059.

A

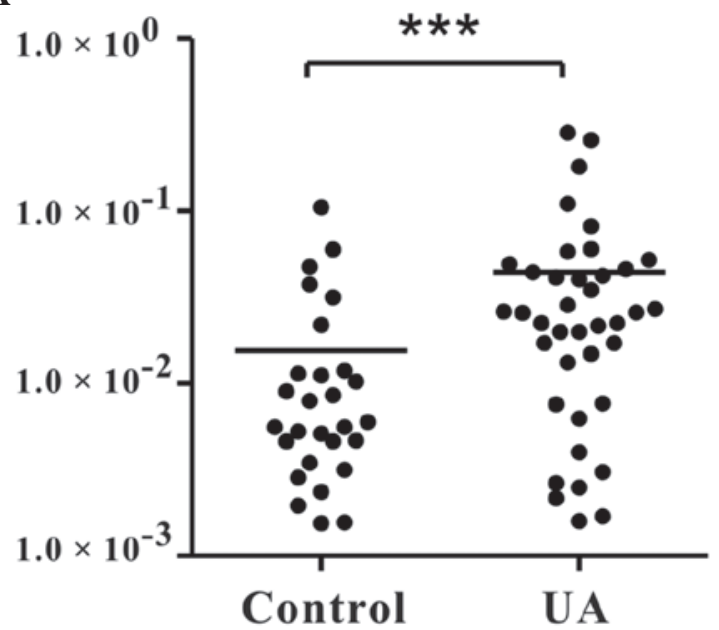

B

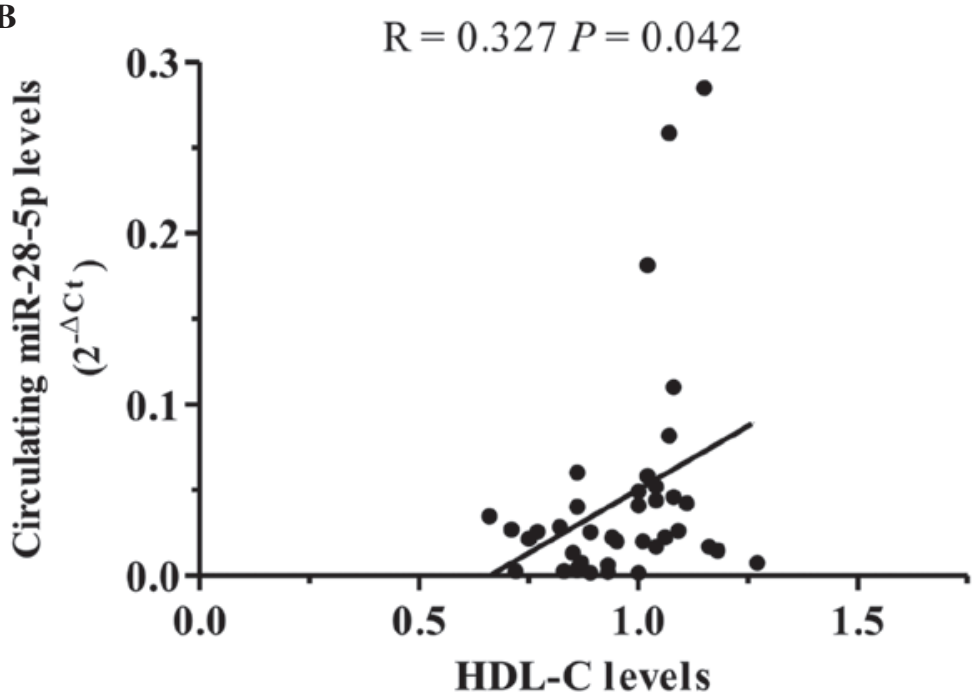

Figure 5. Increased levels of plasma miR-28-5p positively correlate with HDL-C levels. (A) Circulating levels of miR-28-5p in UA and control subjects were determined by reverse transcription-quantitative polymerase chain reaction. The cohort included 28 control subjects and 39 patients with UA. (B) Circulating levels of miR-28-5p positively correlated with HDL-C levels in patients with UA. miR-28-5p, microRNA-28-5p; HDL-C, high-density lipoprotein cholesterol; UA, unstable angina.

increased ABCA1 expression in HepG2 cells. However, the mechanism of miR-28-5p-mediated ABCA1 upregulation and its association with the clinical parameters of cardiovascular disease remains unclear. In the current study, the translational inhibition of ERK2 by miR-28-5p in HepG2 cells was identified, and miR-28-5p was observed to upregulate the expression of ABCA1 via the inhibition of ERK2. Conversely, inhibiting miR-28-5p represses the upregulation of ABCA1 induced by the ERK2 inhibitor, PD98059.
A number of miRNAs including miR-33a/b, miR-758 and miR-144 have been demonstrated to serve important roles in the LXR-mediated ABCA1 pathway by post-transcriptional inhibitory regulation (25-27). These miRNAs inhibit ABCA1 expression through binding to its 3'UTR; however, certain miRNAs, such as miR-122 and miR-370, have been reported to upregulate ABCA1 expression (25-27). Antagonism of miR-122 in mice resulted in the sustained reduction of total plasma cholesterol levels (28). In addition, miR-370 has been 
Table II. Predicted microRNA sites according to mRNA selected regions.

\begin{tabular}{|c|c|c|c|c|}
\hline MicroRNA & StemLoopID & Seed length & P-value & Sites \\
\hline hsa-miR-28-5p & hsa-mir-28 & 16 & 0.0000 & 2 \\
\hline hsa-miR-220c & hsa-mir-220c & 11 & 0.0011 & 2 \\
\hline hsa-miR-297 & hsa-mir-297 & 11 & 0.0011 & 2 \\
\hline hsa-miR-452* & hsa-mir-452 & 10 & 0.0044 & 2 \\
\hline hsa-miR-320c & hsa-mir-320c-1 & 10 & 0.0044 & 4 \\
\hline hsa-miR-320d & hsa-mir-320d-2 & 10 & 0.0044 & 3 \\
\hline hsa-miR-320b & hsa-mir-320b-2 & 10 & 0.0044 & 4 \\
\hline hsa-miR-320a & hsa-mir-320a & 10 & 0.0044 & 2 \\
\hline hsa-miR-628-5p & hsa-mir-628 & 10 & 0.0044 & \\
\hline hsa-miR-1183 & hsa-mir-1183 & 9 & 0.0174 & \\
\hline hsa-miR-181a & hsa-mir-181a-2 & 9 & 0.0174 & 2 \\
\hline hsa-miR-1229 & hsa-mir-1229 & 9 & 0.0174 & 2 \\
\hline hsa-miR-181c & hsa-mir-181c & 9 & 0.0174 & \\
\hline hsa-miR-196a* & hsa-mir-196a-2 & 9 & 0.0174 & \\
\hline hsa-miR-491-5p & hsa-mir-491 & 9 & 0.0174 & \\
\hline hsa-miR-5481 & hsa-mir-5481 & 9 & 0.0174 & \\
\hline hsa-miR-1914 & hsa-mir-1914 & 9 & 0.0174 & \\
\hline hsa-miR-493 & hsa-mir-493 & 9 & 0.0174 & \\
\hline hsa-miR-1250 & hsa-mir-1250 & 9 & 0.0174 & \\
\hline hsa-miR-195* & hsa-mir-195 & 9 & 0.0174 & \\
\hline hsa-miR-497 & hsa-mir-497 & 9 & 0.0174 & \\
\hline hsa-miR-300 & hsa-mir-300 & 9 & 0.0174 & \\
\hline hsa-miR-1255a & hsa-mir-1255a & 9 & 0.0174 & \\
\hline hsa-miR-603 & hsa-mir-603 & 9 & 0.0174 & \\
\hline hsa-miR-1269 & hsa-mir-1269 & 9 & 0.0174 & \\
\hline hsa-miR-1252 & hsa-mir-1252 & 9 & 0.0174 & \\
\hline hsa-miR-302b* & hsa-mir-302b & 9 & 0.0174 & \\
\hline hsa-miR-628-5p & hsa-mir-628 & 9 & 0.0174 & \\
\hline hsa-miR-541* & hsa-mir-541 & 9 & 0.0174 & \\
\hline hsa-miR-22 & hsa-mir-22 & 9 & 0.0174 & \\
\hline
\end{tabular}

observed to directly regulate lipid metabolism via increasing miR-122 levels (28). The current study indicates that blockage of miR-28-5p with its inhibitor reduced ABCA1 protein levels in HepG2 cells, whilst miR-28-5p mimics resulted in the upregulation of ABCA1, suggesting that miR-28-5p is a modulator miRNA for ABCA1 expression.

Numerous studies have described RNA activation, which is the process by which miRNAs are suggested to activate single or multiple genes via the inhibition of negative regulators of certain genes (29-31). ERK2 is an important signaling molecule regulating cellular proliferation and differentiation (12). The biological functions of ERK2 in the cardiac system focus on cardiac development, hypertrophy and protection (32), whereas the role of ERK2 in atherosclerosis predominantly mediates macrophage cholesterol efflux (14). This is based on evidence that the inhibition of ERK2 by PD098059 induces ABCA1 upregulation (14). The current study demonstrated that transfection with miR-28-5p mimics reduced ERK2 expression levels in HepG2 cells, whilst blockage of miR-28-5p with its inhibitor increased ERK2 expression levels. Consequently, the effects of miR-28-5p on ERK2-mediated ABCA1 regulation were observed. ERK2 inhibition antagonizes miR-28-5p inhibitor-mediated ABCA1 reduction. Taken together, these data indicate that the miR-28-5p-mediated regulation of ABCA1 occurred via the inhibition of ERK2, indicating a novel mechanism of the miRNA-associated cholesterol metabolism.

The use of miRNAs in the diagnosis and treatment of cardiovascular disease is an innovative field, with potential strategies including the detection of circulating miRNA levels and the delivery of miRNA inhibitors or mimics (33). The current study indicates that elevated levels of miR-28-5p in plasma are positively correlated with serum HDL-C levels in patients with UA, supporting the role of miR-28-5p-mediated ABCA1 upregulation in the atherosclerotic progress. In the treatment of cardiovascular diseases, there are currently no specific drugs widely available that are able to raise HDL levels efficiently. The use of anti-miRs against ABCA1-associated miRs, such as miR-33 and miR-144, have been confirmed to be potentially therapeutic in the attenuation of the atherosclerotic progress in murine models of atherosclerosis $(25,27)$. The anti-atherogenic properties of ABCA1 have 
been well-studied, with overexpression of ABCA1 in animal models demonstrated to reduce total cholesterol levels and attenuate atherosclerosis $(34,35)$, suggesting that ABCA1 may be a candidate therapeutic target to raise HDL-C levels. The current study confirmed that miR-28-5p, as the first positive modulator of ABCA1 via the inhibition of ERK2, may be a potential target for the treatment of cardiovascular disease. The top 30 predicted and 6 validated miRNAs targeted to ERK 2 are presented in Table II. These miRs have potential roles in the ERK2-mediated ABCA1 upregulation and are suggested to act as agents which raise HDL-C levels to relieve atherosclerotic plaques.

In conclusion, the current study demonstrated that miR-28-5p mediated the upregulation of ABCA1 through the inhibition of ERK2, extending the role of ERK2 in activating cholesterol trafficking. The correlation between circulating miR-28-5p and HDL-C levels in patients with UA suggests that miR-28-5p may participate in the development of atherosclerotic plaques. These data provide novel insight into the cholesterol metabolism and cardiovascular disease. Follow-up studies on the correlation of miR-28-5p with the pathophysiological alterations in patients with UA will lead to an in-depth understanding of the clinical significance and potential use in the diagnosis, treatment and prognosis of patients with UA. In addition, studies in animal models will aid in the elucidation of the biological significance of the miR-28-5p-ERK2-ABCA1 signaling pathway in UA.

\section{Acknowledgements}

This study was supported by the Medical Research Foundation of the Health Planning Commission of Hebei Province of China (grant no. 20130315) and the Scientific Research Program for Returned Scholars, Department of Human Resources and Social Security of Hebei Province of China.

\section{References}

1. Bartel DP: MicroRNAs: Genomics, biogenesis, mechanism, and function. Cell 116: 281-297, 2004.

2. Calin GA and Croce CM: MicroRNA signatures in human cancers. Nat Rev Cancer 6: 857-866, 2006.

3. Ambros V, Lee RC, Lavanway A, Williams PT and Jewell D: MicroRNAs and other tiny endogenous RNAs in C. elegans. Curr Biol 13: 807-818, 2003.

4. Lin SL, Miller JD and Ying SY: Intronic microRNA (miRNA). J Biomed Biotechnol 2006: 26818, 2006.

5. Hooper CL, Dash PR and Boateng SY: Lipoma preferred partner is a mechanosensitive protein regulated by nitric oxide in the heart. FEBS Open Bio 2: 135-144, 2012.

6. Jin L, Hastings NE, Blackman BR and Somlyo AV: Mechanical properties of the extracellular matrix alter expression of smooth muscle protein LPP and its partner palladin; relationship to early atherosclerosis and vascular injury. J Muscle Res Cell Motil 30: 41-55, 2009.

7. Gorenne I, Jin L, Yoshida T, Sanders JM, Sarembock IJ, Owens GK, Somlyo AP and Somlyo AV: LPP expression during in vitro smooth muscle differentiation and stent-induced vascular injury. Circ Res 98: 378-385, 2006.

8. Almeida MI, Nicoloso MS, Zeng L, Ivan C, Spizzo R, Gafà R, Xiao L, Zhang X, Vannini I, Fanini F, et al: Strand-specific miR-28-5p and miR-28-3p have distinct effects in colorectal cancer cells. Gastroenterology 142: 886-896.e9, 2012.

9. Yang M, Yao Y, Eades G, Zhang Y and Zhou Q: MiR-28 regulates Nrf2 expression through a Keap1-independent mechanism. Breast Cancer Res Treat 129: 983-991, 2011.
10. Liu J, Liu Y, Sun YN, Li S, Liu XQ, Li J, Li CM, Tian W, Zhou YT and Shang XM: miR-28-5p involved in LXR-ABCA1 pathway is increased in the plasma of unstable angina patients. Heat Lung Circ 24: 724-730, 2015.

11. Sebolt-Leopold JS and Herrera R: Targeting the mitogen-activated protein kinase cascade to treat cancer. Nat Rev Cancer 4: 937-947, 2004.

12. Pearson G, Robinson F, Beers Gibson T, Xu BE, Karandikar M, Berman K and Cobb MH: Mitogen-activated protein (MAP) kinase pathways: Regulation and physiological functions. Endocr Rev 22: 153-183, 2001.

13. Wang Y: Mitogen-activated protein kinases in heart development and diseases. Circulation 116: 1413-1423, 2007.

14. Zhou X, Yin Z, Guo X, Hajjar DP and Han J: Inhibition of ERK1/2 and activation of liver $X$ receptor synergistically induce macrophage ABCA1 expression and cholesterol efflux. J Biol Chem 285: 6316-6326, 2010.

15. Tall AR: Cholesterol efflux pathways and other potential mechanisms involved in the athero-protective effect of high density lipoproteins. J Intern Med 263: 256-273, 2008.

16. Tall AR, Yvan-Charvet L, Terasaka N, Pagler T and Wang N: HDL, ABC transporters, and cholesterol efflux: Implications for the treatment of atherosclerosis. Cell Metab 7: 365-375, 2008.

17. Bodzioch M, Orsó E, Klucken J, Langmann T, Böttcher A, Diederich W, Drobnik W, Barlage S, Büchler C, Porsch-Ozcürümez $\mathrm{M}$, et al: The gene encoding ATP-binding cassette transporter 1 is mutated in Tangier disease. Nat Genet 22: 347-351, 1999.

18. Brooks-Wilson A, Marcil M, Clee SM, Zhang LH, Roomp K, van Dam M, Yu L, Brewer C, Collins JA, Molhuizen HO, et al: Mutations in ABC1 in Tangier disease and familial high-density lipoprotein deficiency. Nat Genet 22: 336-345, 1999.

19. Rust S, Rosier M, Funke H, Real J, Amoura Z, Piette JC, Deleuze JF, Brewer HB, Duverger N, Denèfle P and Assmann G: Tangier disease is caused by mutations in the gene encoding ATP-binding cassette transporter 1. Nat Genet 22: 352-355, 1999.

20. Dweep H, Sticht C, Pandey P and Gretz N: miRWalk-database: Prediction of possible miRNA binding sites by "walking" the genes of three genomes. J Biomed Inform 44: 839-847, 2011.

21. Girardot M, Pecquet C, Boukour S, Knoops L, Ferrant A, Vainchenker W, Giraudier S and Constantinescu SN: miR-28 is a thrombopoietin receptor targeting microRNA detected in a fraction of myeloproliferative neoplasm patient platelets. Blood 116: 437-445, 2010.

22. Anderson JL, Adams CD, Antman EM, Bridges CR, Califf RM, Casey DE Jr, Chavey WE II, Fesmire FM, Hochman JS, Levin TN, et al; American College of Cardiology; American Heart Association Task Force on Practice Guidelines (Writing Committee to Revise the 2002 Guidelines for the Management of Patients With Unstable Angina/Non-ST-Elevation Myocardial Infarction); American College of Emergency Physicians; Society for Cardiovascular Angiography and Interventions; Society of Thoracic Surgeons; American Association of Cardiovascular and Pulmonary Rehabilitation; Society for Academic Emergency Medicine: ACC/AHA 2007 guidelines for the management of patients with unstable angina/non-ST-Elevation myocardial infarction: A report of the American College of Cardiology/American Heart Association Task Force on Practice Guidelines (Writing Committee to Revise the 2002 Guidelines for the Management of Patients With Unstable Angina/Non-ST-Elevation Myocardial Infarction) developed in collaboration with the American College of Emergency Physicians, the Society for Cardiovascular Angiography and Interventions, and the Society of Thoracic Surgeons endorsed by the American Association of Cardiovascular and Pulmonary Rehabilitation and the Society for Academic Emergency Medicine. J Am Coll Cardiol 50: e1-e157, 2007.

23. Mestdagh P, Van Vlierberghe P, De Weer A, Muth D, Westermann F, Speleman F and Vandesompele J: A novel and universal method for microRNA RT-qPCR data normalization. Genome Biol 10: R64, 2009.

24. Schwindt H, Akasaka T, Zühlke-Jenisch R, Hans V, Schaller C, Klapper W, Dyer MJS, Siebert R and Deckert M: Chromosomal translocations fusing the BCL6 gene to different partner loci are recurrent in primary central nervous system lymphoma and may be associated with aberrant somatic hypermutation or defective class switch recombination. J Neuropathol Exp Neurol 65: 776-782, 2006. 
25. Rayner KJ, Sheedy FJ, Esau CC, Hussain FN, Temel RE, Parathath S, van Gils JM, Rayner AJ, Chang AN, Suarez Y, et al: Antagonism of miR-33 in mice promotes reverse cholesterol transport and regression of atherosclerosis. J Clin Invest 121: 2921-2931, 2011

26. Ramirez CM, Dávalos A, Goedeke L, Salerno AG, Warrier N, Cirera-Salinas D, Suárez Y and Fernández-Hernando C: MicroRNA-758 regulates cholesterol efflux through posttranscriptional repression of ATP-binding cassette transporter A1. Arterioscler Thromb Vasc Biol 31: 2707-2714, 2011.

27. Ramírez CM, Rotllan N, Vlassov AV, Dávalos A, Li M, Goedeke L, Aranda JF, Cirera-Salinas D, Araldi E, Salerno A, et al: Control of cholesterol metabolism and plasma high-density lipoprotein levels by microRNA-144. Circ Res 112: 1592-1601, 2013.

28. Fernández-Hernando C, Ramírez CM, Goedeke L and Suárez Y: MicroRNAs in metabolic disease. Arterioscler Thromb Vasc Biol 33 178-185, 2013.

29. Park SY, Lee JH, Ha M, Nam JW and Kim VN: miR-29 miRNAs activate $\mathrm{p} 53$ by targeting $\mathrm{p} 85 \alpha$ and CDC42. Nat Struct Mol Biol 16 $23-29,2009$

30. Liu P and Wilson MJ: miR-520c and miR-373 upregulate MMP9 expression by targeting mTOR and SIRT1, and activate the $\mathrm{Ras} / \mathrm{Raf} / \mathrm{MEK} /$ Erk signaling pathway and NF-kB factor in human fibrosarcoma cells. J Cell Physiol 227: 867-876, 2012.
31. Cai J, Guan H, Fang L, Yang Y, Zhu X, Yuan J, Wu J and Li M: MicroRNA-374a activates Wnt $/ \beta$-catenin signaling to promote breast cancer metastasis. J Clin Invest 123: 566-579, 2013.

32. Xiang P, Deng HY, Li K, Huang GY, Chen Y, Tu L, Ng PC, Pong NH, Zhao H, Zhang L and Sung RY: Dexrazoxane protects against doxorubicin-induced cardiomyopathy: Upregulation of Akt and Erk phosphorylation in a rat model. Cancer Chemother Pharmacol 63: 343-349, 2009.

33. Dangwal S, Bang C and Thum T: Novel techniques and targets in cardiovascular microRNA research. Cardiovasc Res 93: 545-554, 2012

34. Joyce CW, Amar MJ, Lambert G, Vaisman BL, Paigen B, Najib-Fruchart J, Hoyt RF Jr, Neufeld ED, Remaley AT, Fredrickson DS, et al: The ATP binding cassette transporter A1 (ABCA1) modulates the development of aortic atherosclerosis in C57BL/6 and apoE-knockout mice. Proc Nat Acad Sci USA 99: 407-412, 2002.

35. Cavelier LB, Qiu Y, Bielicki JK, Afzal V, Cheng JF and Rubin EM: Regulation and activity of the human ABCA1 gene in transgenic mice. J Biol Chem 276: 18046-18051, 2001. 